\title{
DEPARTAMENTO DE LASER
}

\section{PO160 Tratamento da discromia com luz intensa pulsada}

\author{
AUTORES: Tamura BM, Tamura P, Cucé LC, Chehin FB, Barreto EA. \\ INSTITUIÇÃO: Universidade de Santo Amaro - São Paulo, SP
}

INTRODução: Existem diversas classificações e etiologias para as discromias com hipopigmentação. Porém na nossa prática de consultório encontramos aqueles casos que a biópsia da área comprometida não esclarece o diagnóstico porque muitas vezes não apresenta qualquer alteração. Alguns pacientes sem diagnóstico específico para classificação têm como história: a pitiríase versicolor tratada anteriormente sem melhora da hipopigmentação e que a cada surto vai apresentando mais algumas lesões; outros que o médico sus peite de hipocromia residual à farmacodermia sem identificação do agente; ou sem etiologia desconhecida e sem história familiar de lesões semelhantes para justificar uma genodermatose.

Relato do CASo: Paciente feminina, solteira, 27 anos, com história de pitiríase versicolor de repetição apresenta desde a adolescência áreas de máculas hipocrômicas mal delimitadas e outras coalescentes no tronco superior, abdômen, dorso, região lombar e parte proximal das coxas anterior. Não se conseguiu determinar reação a medicamento, doença infecciosa ou familiar. A biópsia da pele comprometida apresentouse normal. Tratamos a pacientes com Luz Intensa Pulsada com sessões a cada 14 dias, no total de quatro, filtro de $390 \mathrm{~nm}$ com as seguintes fluências: $24,24,27,27 \mathrm{~J} / \mathrm{cm}^{2}$. Foi prescrito viticromin a 0,5\% em creme para ser utilizado uma vez/dia durante os 7 dias antes do tratamento com a luz. Resultados: Iniciamos o tratamento na região lombar com excelente resposta após essas sessões.

Discussão e Conclusão: A paciente ficou emocionada com o resultado que obtivemos. Estava muito constrangida com essas máculas e já havia tentado terapias tópicas sem qualquer resultado e inclusive utilizando auto-bronzeador. A resposta ao tratamento foi surpreendente. A estimulação da pigmentação somente nas áreas comprometidas, a ausência de queimaduras ou piora da coloração da pele normal, a rapidez da resposta à terapia mostraram que esta é mais uma opção de tratamento, porém necessitamos utilizá-lo em outros pacientes para determinarmos qual irá se beneficiar com ele.

\section{PO 161 Tratamento do hemangioma com luz intensa pulsada}

\author{
AUTORES: Tamura B, Cucé LC, Zane TP, Berti TC, laconelli CR. \\ INSTITUIÇÃO: Derpartamento de Dermatologia da Faculdade de Medicina Santo Amaro
}

INIRODução: Os hemangiomas correspondem à multiplicação de vasos bem diferenciados ou proliferação de
células angioblásticas com neoformações vasculares em graus variados de diferenciação. Apresentam cresci-
mento acelerado durante a infância e $70 \%$ involuem espontaneamente até os 7 anos de idade, nunca surgindo
na fase adulta. Clinicamente são muito heterogêneos quanto ao tamanho, forma e taxa de proliferação. Os
casos que não involuem podem ser tratados com medicamentos como corticóides, interferon ou medidas cirúr-
gicas que incluem intervenções maiores ou simples crioterapia, LASER ou a luz intensa pulsada. A luz pulsada
é um tipo de energia luminosa que abrange as ondas de luz entre 400 e 1.200 nanômetros do espectro eletro-
magnético. Trata as lesões vasculares benignas cutâneas mediante o princípio de fototermocoagulação seletiva.
RELATo Do CAso: Paciente L.M.L., 24 anos, feminino, natural de Minas Gerais, procedente de São Paulo,
auxiliar de classe escolar, solteira com pele tipo III. Paciente apresentava uma mácula vinhosa em região
malar, de aproximadamente $10 \mathrm{~cm}$ de diâmetro, plana com áreas infiltradas de bordas bem definidas com
contornos irregulares. A lesão estava ficando mais evidente desde há 12 anos. Piora com a exposição solar
e ao frio. Foi tratada com luz intensa pulsada com filtros de 520,590 e $640 \mathrm{~nm}$ com bons resultados. Discusão e Conclusão: A luz pulsada vem sendo utilizada no tratamento do hemangioma com bons resultados, porém alguns trabal hos vêm mostrando que sua associação com aplicação do laser no final do tratamento pode ser necessária para a obtenção de melhores resultados.

Motivo DA APRESEnTAÇÃo: Demonstrar resultados obtidos com o tratamento da lesão por meio do uso da luz pulsada com diferentes comprimentos de onda. 


\title{
P0162 Fotorejuvenescimento e tratamento da queratose actínica com luz intensa pulsada
}

\author{
AUTORES: Tamura BM, Tamura PT, Cavassini VM, Cucé LC, Zane TP. \\ INSTITUIÇÃO: Universidade de Santo Amaro - São Paulo, SP
}

INTRODUÇÃO: $O$ fotorejuvenescimento com a luz intensa pulsada com diversos aparelhos tem se mostrado muito eficaz. Como a técnica é ainda muito recente e não se conhecem os efeitos a longo prazo, existem algumas recomendações para que não se utilize essa modalidade terapêutica em determinadas situações, como história de câncer de pele, uso de determinadas drogas, doenças com sensibilidade à luz etc.

Relato do CASO: Paciente do sexo feminino de 37 anos apresentou dois tumores de pele que ao exame anátomo-patológico eram carcinomas basocelulares. Pela sua idade e sua doença a paciente desenvolveu um quadro de baixa estima intenso. Apresentava um quadro de envelhecimento da pele importante e queratoses actínicas na região malar e nasal. Decidimos tratar o fotoenvel hecimento com a luz intensa pulsada. Foram seis sessões com utilização do filtro de $640 \mathrm{~nm}\left(18-27 \mathrm{~J} / \mathrm{cm}^{2}\right)$ em todas as sessões, filtro $520 \mathrm{~nm}$ nas três primeiras cuja fluência variou de $18-27 \mathrm{~J} / \mathrm{cm}^{2}$ e nas últimas três sessões filtro de $590 \mathrm{~nm}$ (fluência de $18-28 \mathrm{~J} / \mathrm{cm}^{2}$ ).

RESULTADOS: A representação fotográfica mostra nitidamente a melhora da pele como da auto-estima da paciente. Discussão e Conclusão: Embora estivéssemos tratando o fotoenvelhecimento da pele da paciente, observamos no segundo retorno da paciente melhora importante das queratoses actínicas e por isso mantivemos toda a terapêutica. A paciente está sendo acompanhada há 18 meses com manutenção da qualidade da pele e sem qualquer sinal de recidiva do carcinoma basocelular, aparecimento de novos tumores ou das queratoses actínicas.

\section{PO 163 Fototerapia localizada: tratamento da psoríase com a luz monocromática de excimer de 308nm}

\author{
AUTORES: Metelmann U. \\ INSTITUIÇÃO: Clínica Particular - São Paulo, SP
}

\begin{abstract}
INTRODUÇÃo: A aplicação do excimer laser com $308 \mathrm{~nm}$ no tratamento da psoríase e outras doenças inflamatórias vem sendo demonstrada na literatura mundial há aproximadamente 5 anos, apresentando resultados superiores aos outros tratamentos convencionais, com menor número de sessões, maior tempo de remissão e menos efeitos colaterais. 0 objetivo desta série de casos é avaliar a resposta terapêutica das lesões de psoríase após as aplicações da luz monocromárica.

Relato de Casos: Foram avaliados 20 pacientes com vitiligo, sendo 11 mulheres e 9 homens, com tempo de evolução da doença entre 5 e 15 anos, sem tratamento há pelo menos 2 meses, com doença restrita a $30 \%$ de área corporal. Foram excluídos pacientes com tratamentos concomitantes, doença extensa, com antecedente de insucesso com fototerapia ou patologia que contra-indicasse aplicação de ultravioleta. Os pacientes foram submetidos inicialmente ao cálculo da dose eritematosa mínima, realizado tratamento semanal, durante até 20 sessões consecutivas, foram descontinuados aqueles que apresentaram melhora em tempo inferior as 20 sessões. Foi utilizada a luz monocromática de excimer de $308 \mathrm{~nm}$, aparelho Excilite ${ }^{\circledR}$-DEKA (Florença-Itália). Um paciente interrompeu o tratamento na 12a sessão por evoluir com piora das lesões. Dezenove pacientes apresentaram melhora gradual, com remissão completa em até 13 sessões. Efeitos colaterais observados foram eritema, bolhas, hiperpigmentação e cefaléia.

Dıscussão: 0 tratamento com a luz monocromática mostrou-se bastante efetivo no tratamento da psoríase. Permitiu execução simples, indolor, semanal, com poucos efeitos colaterais comparando-se a outros métodos utilizados freqüentemente.

Motivo dA APRESENTAÇÃo: Apresentação da luz monocromática excimer de $308 \mathrm{~nm}$ como uma nova modalidade terapêutica para o tratamento da psoríase.
\end{abstract}




\title{
PO 164 Utilização terapêutica da luz monocromática de excimer de 308nm
}

\section{AUTORES: Metelmann U. \\ INSTITUIÇÃO: Clínica Particular - São Paulo, SP}

INTRODUÇÃO: A aplicação do excimer laser $308 \mathrm{~nm}$ e da luz monocromática de excimer $308 \mathrm{~nm}$ tem sido bem documentada no tratamento da psoríase. A utilização no tratamento do vitiligo mostra que a resposta é maior em áreas ultravioleta-dependentes e em doenças localizadas, a associação com outros tratamentos permite resultados mais consistentes. Há descrições também da utilização do excimer laser $308 \mathrm{~nm}$ em outras patologias, como micose fungóide, alopecia areata, granuloma anular, líquen plano oral erosivo, esclerodermia, hipopigmentação pós-cirúrgica, dermatite atópica, desidrose entre outras. Esta série de casos tem o objetivo de demonstrar alguns dos tratamentos realizados e suas respostas terapêuticas.

Relato de Casos: Caso A - foram avaliados seis pacientes com alopecia areata em couro cabeludo, sendo quatro mulheres e dois homens, com tempo de evolução da doença entre 1 e 6 meses, sem tratamento há pelo menos 1 mês. Foram submetidos inicialmente ao cálculo da dose eritematosa mínima; realizado tratamento semanal, em sessões consecutivas até remissão completa do quadro. Foi utilizada a luz monocromática de excimer de $308 \mathrm{~nm}$, aparelho Excilite ${ }^{\circledR}$-DEKA (Florença-Itália). A dose por sessão variou entre 150 $\mathrm{J}$ até $850 \mathrm{~J}$. Os primeiros sinais de pilificação iniciaram-se a partir da 2a sessão. Todos os pacientes obtiveram resposta completa em no máximo oito sessões. Não foram observados efeitos colaterais. Caso 2 paciente masculino, 54 anos, com quadro de desidrose em mãos, não respondendo ao tratamento com corticóide. Foi submetido a duas sessões do excimer $308 \mathrm{~nm}$, com ótima resposta e melhora do prurido.

Dıscussão: 0 tratamento com a luz monocromática mostrou-se seguro e eficaz no tratamento da alopecia areata e da desidrose em monoterapia.

Motivo dA APRESENTAÇÃo: Apresentação da luz monocromática excimer de 308 nm como uma alternativa terapêutica para o tratamento da alopecia areata e da desidrose.

\section{PO 165 "Fotorejuvenescimento fotodinâmico": terapia fotodinâmica associada à luz intensa pulsada no rejuvenescimento cutâneo}

\author{
AUTORES: Metelmann U. \\ INSTITUIÇÃO: Clínica Particular - São Paulo, SP
}

INTRODução: A terapia fotodinâmica vem sendo utilizada na dermatologia há al guns anos no tratamento de queratoses actínicas, carcinomas basocelulares, doença de Bowen, verrugas, hiperplasia de glândula sebácea, entre outras. Mais recentemente tem sido indicada no tratamento do fotoenvelhecimento cutâneo associado à aplicação de luz intensa pulsada ou dye laser. 0 objetivo do trabalho é demonstrar a segurança e eficácia na utilização da terapia combinada, avaliando a tolerância, resultados e efeitos colaterias.

Relato de Casos: Seis pacientes com fototipo II-IV Fitzpatrick, idade variando de 50 a 62 anos, com fotoenvelhecimento cutâneo facial, foram submetidas a aplicação do ácido 5 -aminolevulínico a $20 \%$ seguida da aplicação da luz intensa pulsada, como aparelho Photosilk ®-DEKA (Florença-Itália) com filtro de $550 \mathrm{~nm}$ e $850 \mathrm{~nm}$. Foram observados efeitos colaterais transitórios como eritema, edema, formação de crostas. As pacientes apresentaram melhora da textura, das pigmentações, das rítides e das lesões actínicas.

Dıscussão: 0 tratamento combinado 5-ALA e a IPL mostrou-se seguro e eficaz.

Motivo DA APRESENTAÇÃo: Apresentação da aplicação cosmética da terapia fotodinâmica e terapêutica em lesões tumorais não-melanoma.

\section{PO 166 Estudo comparativo entre Nd:YAG 1064nm e a luz intensa pulsada na epilação}

\author{
AUTORES: Metelmann U. \\ INSTITUIÇÃO: Clínica Particular - São Paulo, SP
}

INTRODução: O objetivo é comparar a segurança e eficácia na epilação de pacientes fototipo II-IV de Fitzpatrick, com a aplicação do laser de Nd:YAG $1064 \mathrm{~nm}$ e da luz intensa pulsada, avaliando-se tempo e 
aplicação, dor, eritema, edema, queda dos pêlos, número de aplicações necessárias.

Relato de CAsos: Foram avaliados 15 pacientes com fototipo II-IV de Fitzpatrick, com idade variando entre 1857 anos, sem patologias associadas. Realizada a aplicação em axila, foi convencionado aplicação do Nd-YAG 1064 $\mathrm{nm}$ na axila esquerda e da luz intensa pulsada, filtro $550 \mathrm{~nm}$ em axila direita, com intervalo de 4 semanas. Foi utilizado o aparelho Photosmartpill ®-DEKA (Florença-Itália). A aplicação do IPL mostrou-se rápida, menos dolorosa, com menos eritema, edema, e queda mais precoce dos pêlos. A aplicação com Nd-YAG mostrou-se um pouco mais lenta e mais dolorosa, não tendo significância estatística, apresentando ainda mais eritema e edema. Dıscussão: 0 tratamento com a luz intensa pulsada mostrou-se efetiva e eficaz comparando-se a aplicação com Nd-YAG. O Nd:YAG apresenta como parâmetros de uma boa aplicação o eritema e edema perifoliculares tendo sido estes considerados inerentes ao tratamento realizado.

Motivo dA APRESENTAÇÃo: Comparação entre dois métodos amplamente utilizados pelos dermatologistas.

\title{
DEPARTAMENTO DE MEDICINA CUTÂNEA E INTERNA
}

\section{PO 167 Delírio de parasitose: sucesso terapêutico com pimozide}

\author{
AUTORES: Yuge S, Romanini AM, Silveira-Sousa D, Gonçalves CB, Martelli ACC. \\ INSTITUIÇÃO: Lauro de Souza Lima - Bauru, SP
}

\begin{abstract}
INTRODUÇÃo: Delírio de parasitose é uma doença rara na qual o paciente tem a falsa e fixa idéia de infestação parasitária. A etiologia da maioria dos casos é uma psicose hipocondríaca monossintomática primária, sem doenças de base ou alterações psiquiátricas. Entretanto, há casos secundários a diabetes, deficiências de vitaminas, abuso de drogas, transtornos mentais orgânicos como demência e outros. 0 início ocorre geralmente aos 60 anos, com predomínio de mulheres (2:1). Clinicamente os pacientes podem apresentar exulcerações, principalmente na face extensora dos membros, ou ter exame físico normal. A maioria dos pacientes refere prurido e parestesia, referidas como "sensação de parasita se movendo". Outra atitude freqüente desses pacientes é trazer à consulta os falsos parasitas dentro de uma caixa para serem "analisados", na verdade são escamas cutâneas, crostas e fios de roupas.
\end{abstract}

Relato dos Casos: 1) Mulher, 40 anos, parda, do lar; relatava que ela e seu marido estavam "infestados" por parasitas há 3 meses. Trouxe à consulta os "parasitas" dentro de um frasco com álcool para serem "analisados". Negava doenças de base e outros sintomas gerais. Exame físico geral: normal. Exame dermatológico: exulcerações, medindo 1,5 a $2 \mathrm{~cm}$ de diâmetro, na região mandibular e antebraços. 2) Homem, 73 anos, branco, aposentado. Referia "bicho geográfico" nos pés e face há 3 anos. Antecedente de déficit de memória para fatos recentes. Exame físico: normal. Exames complementares (casos 1 e 2): bioquímica, função tireoidiana e hemograma normais. Ressonância nuclear magnética de crânio (caso 2): redução volumétrica do encéfalo e infartos cerebrais na substância branca. Tratamento: Pimozide $2 \mathrm{mg} /$ dia por 6 meses com melhora significativa.

Dıscussão: 0 primeiro caso envolve delírio de parasitose primário com "folie a deux", isto é, delírio é compartiIhado por outra pessoa, geralmente cônjuge. 0 caso 2 foi diagnosticado como delírio de parasitose secundário a quadro demencial incipiente. A droga de escolha para ambos os casos é o Pimozide, um neuroléptico bloqueador seletivo da recaptação da dopamina. Nos casos secundários deve ser corrigida a doença de base, se possível.

MoTnVo DA APRESENTAÇ̃̃o: Freqüentemente o dermatologista, em vez do psiquiatra, é o primeiro a ser consultado, pois os indivíduos acreditam firmemente que tem uma infestação parasitária e não uma al teração psiquiátrica. Assim, tem sido cada vez mais freqüente a abordagem deste tema na literatura dermatológica, tornando-se necessário o conhecimento do quadro clínico e abordagem terapêutica desta doença psicodermatológica.

\section{PO 168 Tratamento de psoríase artropática e eritrodérmica severa com infliximab}

\author{
AUTORES: Yuge S, Romanini AM, Sarahushi L, Gonçalves CB, Martelli ACC. \\ INSTITUIÇÃO: UNIFESP - Escola Paulista de Medicina - São Paulo, SP
}

INTRODuçÃo: A psoríase artropática é uma doença crônica, heterogênea, de patogenia desconhecida que interfere significativamente na qualidade de vida do paciente. Está incluída no grupo das artropatias soro- 